\title{
Efficacy of Anal Needle Electrodes for Intraoperative Spinal Cord Monitoring with Transcranial Muscle Action Potentials
}

Kazuyoshi Kobayashi ${ }^{1}$, Kei Ando ${ }^{1}$, Hideki Yagi ${ }^{2}, K$ Kenyu Ito ${ }^{1}$, Mikito Tsushima ${ }^{1}$, Masayoshi Morozumi ${ }^{1}$, Satoshi Tanaka ${ }^{1}$, Masaaki Machino ${ }^{1}$, Kyotaro Ota ${ }^{1}$, Yukihiro Matsuyama ${ }^{3}$, Naoki Ishiguro ${ }^{1}$, Shiro $\operatorname{Imagama}^{1}$

${ }^{1}$ Department of Orthopedic Surgery, Nagoya University Graduate School of Medicine, Nagoya, Japan

${ }^{2}$ Department of Orthopaedic Surgery, Japanese Red Cross Nagoya Daiichi Hospital, Nagoya, Japan

${ }^{3}$ Department of Orthopaedic Surgery, Hamamatsu University School of Medicine, Hamamatsu, Japan

Study Design: Retrospective study.

Purpose: To examine the relationship between postoperative bowel bladder disorder (BBD) and the efficacy of needle electrodes for the external anal sphincter (EAS) in intraoperative spinal cord monitoring with transcranial muscle action potentials (Tc-MsEP).

Overview of Literature: Spinal surgery for spina bifida, spinal cord tumor, and spinal tethered cord syndrome has a high rate of postoperative BBD. Monitoring of the EAS with Tc-MsEP is frequently performed during spinal surgery. We initially used plug-surface electrodes for this purpose, but have more recently switched to needle electrodes for the monitoring of the EAS. To date, there has been no comparison between the utility of these electrodes.

Methods: Waveform derivation, exacerbation of postoperative BBD, and sensitivity and specificity for prediction of BBD by $70 \%$ amplitude reduction of EAS activity using needle and plug-surface electrodes were investigated in 239 spine surgeries. The cut-off for the \% drop in amplitude for BBD prediction was determined for EAS monitoring using a needle electrode.

Results: The overall rate of postoperative BBD aggravation was 7.1\% (17/239 cases), with the individual rates using needle and plug-surface electrodes being $6.9 \%(8 / 116)$ and $7.3 \%(9 / 123)$, respectively. The waveform derivation rate was significantly higher using needle electrodes $(91.3 \%$ [106/116] vs. 76.4\% [94/123], $p<0.01)$. In patients with baseline waveform detection, the sensitivity and specificity for postoperative BBD were similar in the two groups. With needle electrodes, a cutoff amplitude of Tc-MsEP for the EAS at the end of surgery of $25 \%$ of the baseline amplitude had a sensitivity of $89 \%$ and specificity of $82 \%$ for the prediction of postoperative BBD aggravation.

Conclusions: The significantly higher waveform derivation rate using needle electrodes suggests that these electrodes are effective for monitoring the EAS in spinal surgery in cases with preoperative BBD.

Keywords: Anal sphincter; Motor evoked potential; Electrode; Motor deficit; Spine

Received Sep 27, 2017; Revised Nov 9, 2017; Accepted Dec 17, 2017

Corresponding author: Shiro Imagama

Department of Orthopedic Surgery, Nagoya University Graduate School of Medicine, 65 Tsurumai Showa-ward, Aichi 466-8550, Japan Tel: +81-52-741-2111, Fax: +81-52-744-2260, E-mail: imagama@med.nagoya-u.ac.jp 


\section{Introduction}

In spinal surgery, postoperative bowel bladder disorder (BBD) occurs at a relatively high rate in patients with conditions such as spina bifida, spinal cord tumor, and spinal tethered cord syndrome. Intraoperative monitoring of the external anal sphincter (EAS) for the prevention of intraoperative neurological deficit was first reported by James et al. [1], and subsequently, there have been many reports on monitoring with transcranial muscle action potentials (Tc-MsEP), including that of the EAS, in spinal surgery [2-16].

We have previously described plug-surface electrodes for the intraoperative monitoring of the EAS, which has a lower derivation rate than other muscles [2]. Based on the possible influence of the electrode type on the derivation rate, we subsequently used needle electrodes for monitoring the EAS with Tc-MsEP. In the current study, we compared the rates of postoperative BBD after intraoperative spinal cord monitoring using needle and plug-surface electrodes and examined the appropriate alarm points for waveform deterioration in EAS monitoring.

\section{Materials and Methods}

\section{Patient selection and neurological evaluation}

At our hospital, 269 consecutive spinal surgeries with intraoperative neurophysiological monitoring with TcMsEP were performed from April 2009 to December 2011. This study was approved by the Institutional Review Board of Nagoya University Hospital (IRB approval no., 354-3) and each patient provided informed consent before enrollment. After the exclusion of 25 reoperation cases and five dialysis patients (preoperative motor status itself was not considered in the exclusion criteria), 239 patients (104 males and 135 females) were included in the study. The mean age of the patients at surgery was 48.5 years (range, $8-86$ years). There were 59 cervical cases (24.7\%), 107 thoracic cases (44.8\%), and 73 lumbar cases (30.5\%); 23 cases (9.6\%) had preoperative BBD. Bladder bowel function was measured using the Japanese Orthopaedic Association (JOA) score before and after the surgery, with a JOA score of $<0$ indicating preoperative $\mathrm{BBD}$ and a decline in a score of $>1$ indicating postoperative BBD aggravation. Among the 239 patients, plug-surface electrodes were used in 123 consecutive patients and
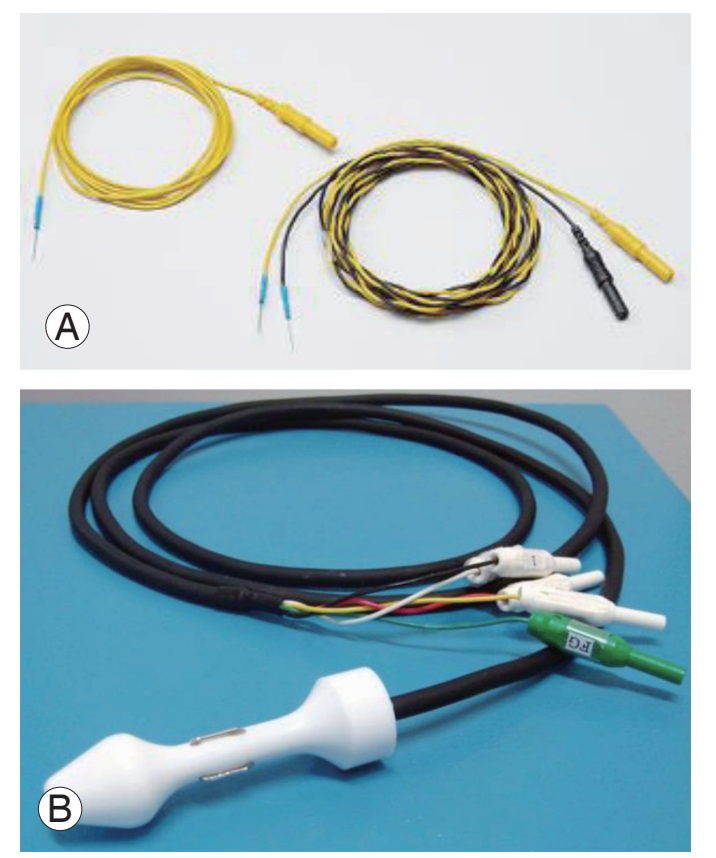

Fig. 1. Two types of electrodes used for the external anal sphincter. (A) Needle electrode. (B) Plug-surface electrode.

needle electrodes were used in 116 consecutive patients for monitoring the EAS with Tc-MsEP (Fig. 1). Details of the two groups are shown in Table 1. Waveform derivation, postoperative BBD aggravation, and sensitivity and specificity for the prediction of BBD aggravation at an amplitude reduction of $70 \%$ were investigated. The cutoff for the amplitude reduction with needle electrodes for the prediction of postoperative BBD aggravation was also examined.

\section{Anesthetic management and general conditions dur- ing surgery}

A minimal benzodiazepine dose was used as a pre-anesthetic to avoid possible suppression of waveform latency and amplitude. Propofol (3-4 mg/kg), fentanyl (2 mg/ $\mathrm{kg})$, and vecuronium $(0.12-0.16 \mathrm{mg} / \mathrm{kg})$ were administered for induction, and anesthesia was maintained with propofol (50-100 $\mu \mathrm{g} / \mathrm{kg} / \mathrm{min})$, fentanyl (1-2.5 $\mu \mathrm{g} / \mathrm{kg} / \mathrm{hr})$, and vecuronium $(0.01-0.04 \mathrm{mg} / \mathrm{kg} / \mathrm{hr})$. Concomitant hypotensive anesthesia was induced as appropriate with continuous prostaglandin $\mathrm{E}_{1}$ and a short-acting $\beta 1$ blocker (landiolol). Patients were maintained in a normothermic state and their temperature was raised in the event of possible intraoperative spinal damage. End-tidal $\mathrm{CO}_{2}$ was maintained in the reference range throughout the surgery. 
Table 1. Characteristics of cases treated using monitoring with needle and plug-surface electrodes

\begin{tabular}{|c|c|c|c|}
\hline Characteristic & Needle $(n=116)$ & Plug-surface (n=123) & $p$-value \\
\hline \multicolumn{4}{|l|}{ Background } \\
\hline Age (yr) & $47.1 \pm 20.3$ & $49.2 \pm 18.3$ & NS \\
\hline Female & 69 & 66 & NS \\
\hline \multicolumn{4}{|l|}{ Region of spine } \\
\hline Cervical & 25 & 34 & NS \\
\hline Thoracic & 53 & 54 & NS \\
\hline Lumbar & 38 & 35 & NS \\
\hline \multicolumn{4}{|l|}{ Diagnosis } \\
\hline Spinal tumor (cervical, thoracic) & $34(29)$ & $33(27)$ & NS \\
\hline Scoliosis & $18(16)$ & $14(11)$ & NS \\
\hline Lumbar degenerative disease & $14(12)$ & $17(14)$ & NS \\
\hline $\begin{array}{l}\text { Ossification of the posterior longitudinal ligament, ossification of the yellow liga- } \\
\text { ment }\end{array}$ & $13(11)$ & $20(16)$ & NS \\
\hline Lumbar intradural extramedullary tumor & $7(6)$ & $11(9)$ & NS \\
\hline Cervical spondylotic myelopathy & $6(5)$ & $5(4)$ & NS \\
\hline Cervical involvement in rheumatoid arthritis & $2(2)$ & $3(2)$ & NS \\
\hline Spinal infection & $3(3)$ & $2(2)$ & NS \\
\hline Spinal cord hernia & $2(2)$ & $2(2)$ & NS \\
\hline Tethered cord syndrome & $1(1)$ & $4(3)$ & NS \\
\hline Others & $16(14)$ & $12(10)$ & NS \\
\hline Preoperative bowel bladder disorder & $10(7)$ & $13(10)$ & NS \\
\hline
\end{tabular}

Values are presented as mean \pm standard deviation or number (\%).

NS, not significant.

For intraoperative body temperature monitoring, a catheter with a vesical temperature sensor was used. Hemodynamic data were electronically recorded with invasive arterial blood pressure (BP) monitoring. Systolic BP variation was measured during the surgery, and systolic BP was determined at the time of waveform deterioration.

\section{Stimulation and recording methods}

An MS120B (Nihon-Kohden, Tokyo, Japan) was used to perform transcranial stimulation, with parameters of 5 stimuli in a row at 2-ms intervals, a constant biphasic current of $200 \mathrm{~mA}$ for $500 \mu \mathrm{s}$, a $50-1000-\mathrm{Hz}$ filter, and a $100-$ ms epoch time with $\leq 20$ recorded signal responses. The stimulated point was $2 \mathrm{~cm}$ anterior and $6 \mathrm{~cm}$ lateral from the $\mathrm{Cz}$ location over the cerebral cortex motor area. Using a Neuromaster MEE-1232 ver. 05.10 (Nihon-Kohden), which is expandable to 32 channels, muscle action potentials were recorded from the upper and lower extremities via a pair of needle electrodes that were 3-5 Tc-MsEPs apart. The bilateral trapezius, triceps, deltoid, biceps, brachioradialis, abductor digit minimi, extensor carpi ulnaris, adductor longus, quadriceps femoris, hamstrings, tibialis anterior, gastrocnemius, and abductor halluces were used as target muscles. From April 2009 to August 2010, we used 2-channel plug-surface electrodes for the EAS for consecutive patients, and from September 2010 to December 2011, we used bilateral pairs of needle electrodes that were $2 \mathrm{~cm}$ apart outside the anus for consecutive patients (Fig. 1). Tc-MsEP data from these muscles were used for analysis.

\section{Statistical analysis}

Significance was assessed using either the Student $t$-test or the Fisher's exact test. Receiver operating characteristic (ROC) curves were used to determine the cutoff amplitude. Sensitivity and specificity at the optimal cutoff values 
were calculated. All $p<0.05$ were considered significant in all analyses. Data analysis was performed with IBM SPSS ver. 22.0 for Windows (IBM Corp., Armonk, NY, USA).

\section{Results}

The waveform derivation rate was significantly higher using needle electrodes $(91.3 \%$ [106/116] versus $76.4 \%$ [94/123], $p<0.01$ ) (Fig. 2). This rate was also significantly higher using needle electrodes in cases with preoperative $\operatorname{BBD}(60 \%[6 / 10]$ versus $16 \%[2 / 13], p<0.05)$ (Fig. 3A) and without preoperative BBD (94.3\% [100/106] versus $83.6 \%$ [92/110], $p<0.05]$ (Fig. 3B). The etiology of postoperative $\mathrm{BBD}$ is shown for cases treated using needle and plugsurface electrodes in Table 2. The incidence of postoperative BBD aggravation was 7.1\% (17/239) in all cases, 6.9\% (8/116) using needle electrodes, and 7.3\% (9/123) using plug-surface electrodes (Table 2).

In patients with baseline waveform detection wherein

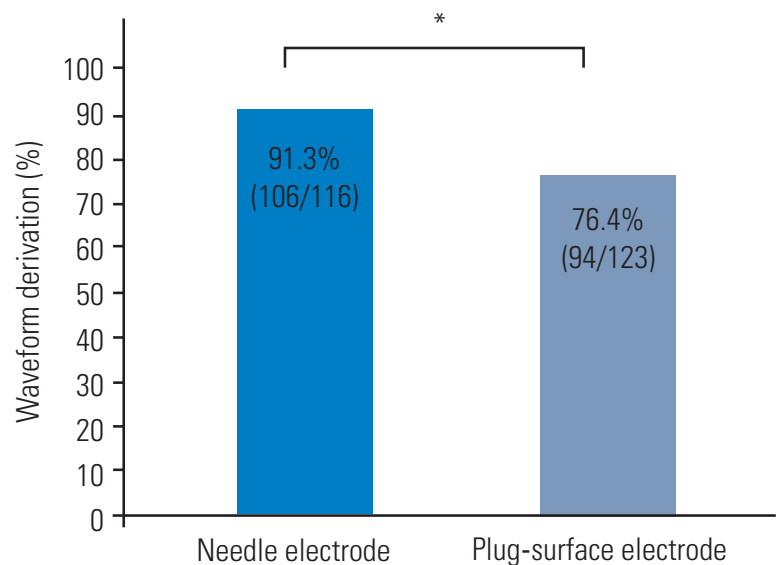

Fig. 2. The waveform derivation rate was significantly higher using the needle electrode (91.3\% [106/116] vs. 76.4\% [94/123]). " $p<0.01$. needle electrodes were used, the sensitivity and specificity for the prediction of BBD aggravation were $88 \%$ and $85 \%$, respectively (Table 3 ). Using plug-surface electrodes, the sensitivity and specificity for the prediction of BBD aggravation were approximately $89 \%$ and $80 \%$, respectively (Table 4). When using needle electrodes for the EAS, the intraoperative Tc-MsEP cutoff amplitude at the end of
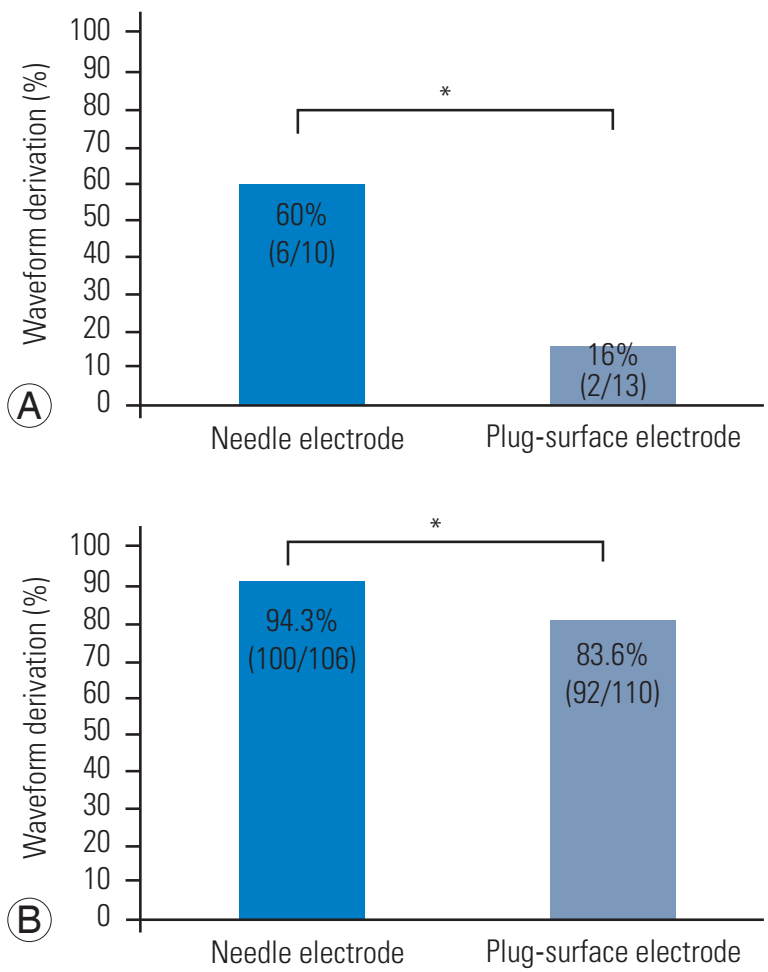

Fig. 3. (A) In a case with preoperative bowel bladder disorder (BBD), the waveform derivation rate was significantly higher using the needle electrode $(60 \%[6 / 10]$ vs. $16 \%[2 / 13]) .{ }^{*} p<0.05$. (B) In a case without preoperative $\mathrm{BBD}$, the waveform derivation rate was also significantly higher using the needle electrode $(94.3 \%$ [100/106] vs. $83.6 \%$ [92/110]). $p<0.05$.

Table 2. Incidence of postoperative bowel bladder disorder aggravation in cases treated using monitoring with needle and plug-surface electrodes

\begin{tabular}{lccc}
\hline Etiology & Needle & Plug-surface & Total \\
\hline Spinal tumor (cervical, thoracic) & $15(5 / 34)$ & $12(4 / 33)$ & $13(9 / 67)$ \\
\hline Ossification of the posterior longitudinal ligament, ossification of the yellow ligament & $23(3 / 13)$ & $10(2 / 20)$ & $15(5 / 33)$ \\
\hline Lumbar intradural extramedullary tumor & $0(0 / 7)$ & $9(1 / 11)$ & $6(1 / 18)$ \\
\hline Tethered cord syndrome & $0(0 / 1)$ & $25(1 / 4)$ & $20(1 / 5)$ \\
\hline Scoliosis (idiopathic, congenital) & $0(0 / 18)$ & $7(1 / 14)$ & $3(1 / 32)$ \\
Others & $0(0 / 43)$ & $0(0 / 45)$ & $0(0 / 88)$ \\
\hline Total & $6.9(8 / 116)$ & $7.3(9 / 123)$ & $7.1(17 / 239)$ \\
\hline
\end{tabular}

Values are presented as \% (number/total number). 
Table 3. Relationship of postoperative BBD with waveform deterioration with needle electrode

\begin{tabular}{lccc} 
& \multicolumn{3}{c}{ Postoperative BBD } \\
\cline { 2 - 4 } Variable & Present & Absent & Total \\
Waveform deterioration $(+)$ & 7 & 15 & 22 \\
Waveform deterioration $(-)$ & 1 & 83 & 84 \\
Total & 8 & 98 & 106 \\
\hline
\end{tabular}

Sensitivity, 88\%; specificity, $85 \%$; false-positive rate, $15 \%$; falsenegative rate, $12 \%$; positive predictive value, $32 \%$; and negative predictive value, $99 \%$.

$\mathrm{BBD}$, bowel bladder disorder.

Table 4. Relationship of postoperative BBD with waveform deterioration with plug-surface electrode

\begin{tabular}{lccc} 
& \multicolumn{3}{c}{ Postoperative BBD } \\
\cline { 2 - 4 } Variable & Present & Absent & Total \\
Waveform deterioration (+) & 8 & 17 & 25 \\
Waveform deterioration (-) & 1 & 68 & 69 \\
Total & 9 & 85 & 94 \\
\hline
\end{tabular}

Sensitivity, $89 \%$; specificity, $80 \%$; false-positive rate, 20\%; falsenegative rate, $11 \%$; positive predictive value, $32 \%$; and negative predictive value, $99 \%$.

$\mathrm{BBD}$, bowel bladder disorder.

surgery in the ROC analysis was $25 \%$ of the baseline amplitude, with a sensitivity of $89 \%$ and specificity of $82 \%$ for the prediction of BBD aggravation (Fig. 4). There were no complications with plug-surface or needle electrodes.

\section{Discussion}

In 1953, Floyd and Walls [3] first reported an electromyogram using a surface electrode placed on the skin of the anus. Monitoring of the EAS with Tc-MsEPs is now widely performed in spinal surgery to prevent intraoperative neurological deficit $[1,4,7-14,16,17]$; in addition, electrophysiological tests for bladder and rectal function have been developed. Many institutions now use monitoring of the EAS with Tc-MsEP to prevent intraoperative paralysis during spinal surgery. The EAS has pudendal innervation, in which the dominant area is S2-4 and is thus useful for intraoperative monitoring of urinary function and dysuria due to spinal diseases [6].

The plug-surface electrode is relatively simple and less invasive, but the waveform derivation rate is low [7]. In

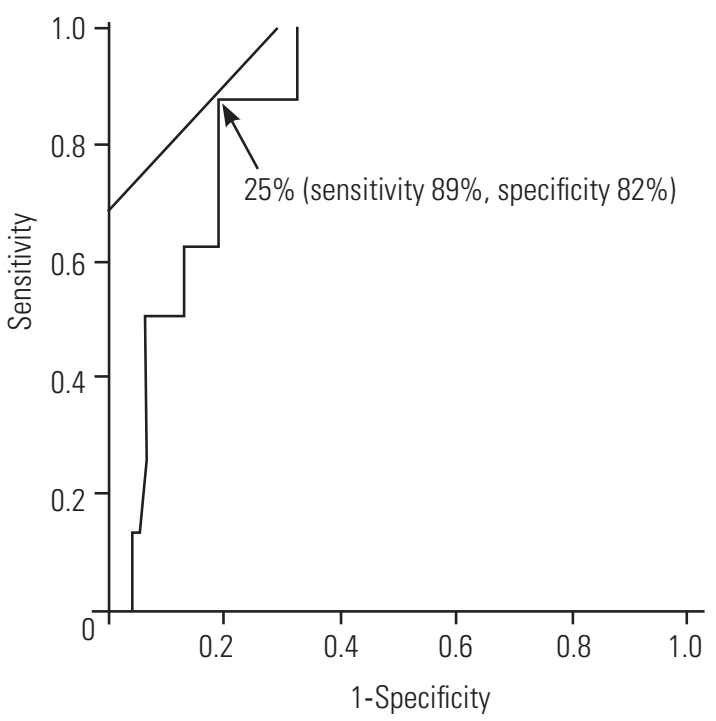

Fig. 4. Receiver operating characteristic curve for the determination of the cutoff for the prediction of postoperative bowel bladder disorder aggravation using the intraoperative transcranial muscle action potentials amplitude (expressed as \% of baseline) obtained for the external anal sphincter using needle electrodes.

our series, the waveform derivation rate from the EAS using plug-surface electrodes was $76.4 \%$, which was significantly lower than the rate of $91.3 \%$ using needle electrodes. The sensitivity and specificity for the prediction of BBD aggravation based on a waveform reduction of $\geq 70 \%$ in monitoring of the EAS with Tc-MsEP were similar for the two electrodes. However, we found that it was difficult to detect baseline waveform using a plug-surface electrode in a case with preoperative BBD; thus, it is preferable to use needle electrodes for the anus. In fact, we have always used needle electrodes for all extremities.

Muramoto et al. $[8,13]$ reported cutoff values for lower limb muscles concerning paralysis exacerbation in spinal thoracic surgery, but there have been no reports on cutoff values for intraoperative monitoring with regard to postoperative $\mathrm{BBD}$. In our study, ROC analysis using needle electrodes for monitoring of the EAS with Tc-MsEP gave a cutoff of $25 \%$ of the baseline amplitude for the prediction of postoperative $\mathrm{BBD}$ aggravation. A waveform amplitude reduction of $70 \%$ in spinal surgery is the recommended criterion for an alarm point [12], and an alarm point of $70 \%$ waveform reduction may also be appropriate for the EAS. However, there are many false-positive cases at the $70 \%$ alarm point, and further study is needed to improve the accuracy of the monitoring. Specifically, there is a need to evaluate the absolute amplitude and to consider 
both single-train and double-train stimulation methods.

In patients with baseline waveform detection, the sensitivity and specificity for the prediction of $\mathrm{BBD}$ aggravation did not differ significantly between the two electrodes. However, deeper muscles could be detected using needle electrodes, and this resulted in a better waveform derivation rate and no complications. Therefore, we consider needle electrodes to be the most useful for monitoring $\mathrm{BBD}$, although this may be difficult in patients with injuries that might be caused by needle insertion, as well as in patients with hemorrhoids.

\section{Conclusions}

In patients with baseline waveform detection, sensitivity and specificity were equivalent for needle and plugsurface electrodes; however, the waveform derivation rate was significantly higher with needle electrodes. The study provides the first report of a cutoff value of $25 \%$ for the prediction of postoperative BBD aggravation using needle electrodes and shows that needle electrodes are effective for monitoring the EAS muscles during spinal surgery, particularly in cases with preoperative BBD.

\section{Conflict of Interest}

No potential conflict of interest relevant to this article was reported. Funding was from institutional sources only.

\section{Author Contributions}

Kobayashi $\mathrm{K}$ analyzed data and wrote the manuscript; Ando K, Yagi Y, Ito K, Tsushima M, Morozumi M, Tanaka S, Machino M, Ota K, Matsuyama Y, Ishiguro N, and Imagama $S$ designed the study and collected data.

\section{References}

1. James HE, Mulcahy JJ, Walsh JW, Kaplan GW. Use of anal sphincter electromyography during operations on the conus medullaris and sacral nerve roots. Neurosurgery 1979;4:521-3.

2. Kobayashi K, Ando K, Yagi H, et al. Prevention and prediction of postoperative bowel bladder disorder using an anal plug electrode with Tc-MsEP monitoring during spine surgery. Nagoya J Med Sci 2017;79:459-66.
3. Floyd WF, Walls EW. Electromyography of the sphincter ani externus in man. J Physiol 1953;122:599-609.

4. Haghighi SS, Zhang R. Activation of the external anal and urethral sphincter muscles by repetitive transcranial cortical stimulation during spine surgery. J Clin Monit Comput 2004;18:1-5.

5. Wiesner A, Jost WH. EMG of the external anal sphincter: needle is superior to surface electrode. Dis Colon Rectum 2000;43:116-8.

6. Kumar GS, Rajshekhar V, Babu KS. Intraoperative mapping of sacral nervous system (S2-4). Br J Neurosurg 2006;20:396-402.

7. Inoue S, Kawaguchi M, Takashi S, et al. Intraoperative monitoring of myogenic motor-evoked potentials from the external anal sphincter muscle to transcranial electrical stimulation. Spine (Phila Pa 1976) 2002;27:E454-9.

8. Muramoto A, Imagama S, Ito Z, et al. The cutoff amplitude of transcranial motor-evoked potentials for predicting postoperative motor deficits in thoracic spine surgery. Spine (Phila Pa 1976) 2013;38:E21-7.

9. Ito Z, Matsuyama Y, Ando M, et al. What is the best multimodality combination for intraoperative spinal cord monitoring of motor function?: a multicenter study by the Monitoring Committee of the Japanese Society for Spine Surgery and Related Research. Global Spine J 2016;6:234-41.

10. Teramoto T. Functional characteristics features in anatomy and physiology of the anal canal. Nichisyougekaishi 1990;23:2147-50.

11. Kothbauer KF, Deletis V. Intraoperative neurophysiology of the conus medullaris and cauda equina. Childs Nerv Syst 2010;26:247-53.

12. Ito Z, Imagama S, Sakai Y, et al. A new criterion for the alarm point for compound muscle action potentials. J Neurosurg Spine 2012;17:348-56.

13. Muramoto A, Imagama $S$, Ito $Z$, et al. The cutoff amplitude of transcranial motor evoked potentials for transient postoperative motor deficits in intramedullary spinal cord tumor surgery. Spine (Phila Pa 1976) 2014;39:E1086-94.

14. Kobayashi K, Imagama S, Ito Z, et al. Transcranial motor evoked potential waveform changes in corrective fusion for adolescent idiopathic scoliosis. J Neurosurg Pediatr 2017;19:108-15.

15. Nandedkar SD, Sanders DB, Stalberg EV, Andreassen S. Simulation of concentric needle EMG motor unit 
action potentials. Muscle Nerve 1988;11:151-9.

16. Bischoff C, Stalberg E, Falck B, Eeg-Olofsson KE. Reference values of motor unit action potentials obtained with multi-MUAP analysis. Muscle Nerve 1994;17:842-51.
17. Kobayashi K, Imagama S, Ito Z, Ando K, Hida T, Ishiguro N. Prevention of spinal cord injury using brain-evoked muscle-action potential ( $\mathrm{Br}(\mathrm{E})-\mathrm{MsEP})$ monitoring in cervical spinal screw fixation. Eur Spine J 2017;26:1154-61. 